\title{
Why HIV Patients Are Not Adherent to Antiretroviral Therapy: A Study Among HIV Patients Attending Anti-Retroviral Therapy Centre in A Tertiary Care Hospital in Kolkata, West Bengal
}

\author{
Dr. Aditi Chaudhuri ${ }^{1}$,Dr. Sita Chattopadhyay ${ }^{2}$, Dr. Raghunath Misra ${ }^{3}$, Dr. \\ Rupali Ganguli ${ }^{4}$, \\ ${ }^{I}$ Assistant Professor, Department of Community Medicine, Institute of Post Graduate Medical \\ Education \& Research, Kolkata. \\ ${ }^{2}$ Associate Professor, Department of Community Medicine, Institute of Post Graduate Medical \\ Education \& Research, Kolkata. \\ ${ }^{3}$ Professor, Department of Community Medicine, Institute of Post Graduate Medical \\ Education \& Research, Kolkata. \\ ${ }^{4}$ Senior Medical Officer, ART Centre, Institute of Post Graduate Medical Education \\ \& Research, Kolkata.
}

\begin{abstract}
Background \& objectives: The introduction of ART therapy has been able to improve the survival of HIV infected individuals but non-adherence to ART is a major cause of HIV drug resistance. Globally around 33\% to $38 \%$ of HIV infected adults do not adhere to treatment. However, there is a dearth of research in India in understanding the reasons for non adherence to ART regimen. The present study was conducted at the ART centre of tertiary care hospital in West Bengal, with the objectives to study the sociodemographic and clinical profile of the patients, to estimate the prevalence of non adherence and to assess the causes of non adherence to ART regimen among the study population. Methods: The study was both retrospective \& prospective, observational with cross-sectional design. 400 Patient Treatment cards were reviewed to collect data on socioclinical profile and nonadherence. In the next phase the nonadherent persons were interviewed to find out the cause of nonadherence. Result: 26.5\% of the study population were not adherent to ART therapy. Constraints such as forgetfulness to take the medications, economic reason, hopelessness about cure(11.32\%) etc undermine their intentions to adhere to ART regimen. Conclusion: Efforts are required at the management as well as implementation level for constant regular follow-up \& motivation of all patients under ART with counseling.
\end{abstract}

Keywords: ART, socio demographic, adherence, causes

\section{Introduction}

Acquired Immunodeficiency Syndrome (AIDS) has long been considered a serious public health problem in both developed and developing countries. Globally, 35.3 million people were living with HIV in 2012 which is an increase from previous years as more people are receiving the life-saving antiretroviral therapy. The UNAIDS report 2013 recorded 33\% decline in the number of new infections from 2001[1]. In 1986, when the first known case of HIV was diagnosed by Dr. Suniti Solomon and her associate Dr. Sellappan Nirmala amongst female sex workers in Chennai, Tamil Nadu [2] probably nobody could predict that within next 3 decades India will be home to the world's third-largest population suffering from HIV/AIDS (following South Africa and Nigeria). National adult (15-49 years) HIV prevalence is estimated at $0.26 \%$ $(0.22 \%-0.32 \%)$ in 2015 [3] with majority of reported cases occurring in sexually active and economically productive age group 15-49 years. At the end of the 1980s, a rapid spread of HIV was observed among injecting drug users in Manipur, Mizoram and Nagaland [4]. Death of young adults made damaging impacts on the economy of the society.

The triple-drug antiretroviral therapy for HIV, launched in 1996 during World AIDS conference in Vancouver literally changed the life of the victims. So far no other disease in the history of mankind has attracted so much money and attention. The introduction of therapy (ART) has been able to improve the survival of HIV infected individuals by inhibiting viral replication and delaying the progression to AIDS [5]. Earlier initiation of highly active antiretroviral therapy (HAART) at CD4 counts about 350 cells/mm3 in HIV-infected patients decreased their likelihood of transmitting the virus to partners by 96 per cent $[6,7]$. Greater than $95 \%$ adherence has been suggested for the success of ART. Additionally, non-adherence to ART is a major cause of HIV drug resistance. However, literature review suggests that globally around 33\% to 38\% of HIV infected adults do not adhere to treatment regimens of ART [8].Studies on ART adherence in India, have shown varying 
estimates of ART adherence (44\%-86\%) [9]. However, there is a dearth of research in India in understanding the reasons for non adherence to ART regimen.

With the above background, our present study was conducted at the ART centre of I.P.G.M.E \&R. and S.S.K.M. Hospital, a major tertiary care hospital in Kolkata, West Bengal, with the following objectives:

1.To study the sociodemographic and clinical profile of the patients attending ART centre at S.S.K.M. Hospital, Kolkata.

2.To estimate the prevalence of non adherence to ART regimen among the study population.

3.To assess the causes of non adherence among the study population.

\section{Methods And Materials}

The present study was both retrospective \& prospective, observational with cross-sectional design conducted at Anti Retroviral Treatment (ART) Centre of I.P.G.M.E \&R. and SSKM Hospital, Kolkata between May to June 2014. Study population was selected from the cohort of all HIV patients registered from December 2012 till $31^{\text {st }}$ January 2014 i.e. at least 3 months prior to the commencement of our study (December 2012 being the time when the ART centre became functional and enrolment of the first patient occurred). An interval of 3 months was required to estimate nonadherence as according to NACO guidelines, supply of ARV to a newly enrolled patient is provided for 15 days at first and then every monthly a follow up visit is required. Services at the ART centre included integrated medical services for the treatment of HIV and related illnesses, prevention programs, and nutrition counselling, adherence and risk reduction counselling at each clinic visit following initiation of ART. During the study period, total number of patients registered at the ART Centre of SSKM Hospital was 863 out of which only 744 were found to be eligible for ART treatment. Out of the 744 eligible for treatment only 667 were receiving outpatient HIV clinical care (77 were lost to follow up or died or went to another centre). We estimated a sample size of 400 considering prevalence of $50 \%$ for treatment adherence with a $95 \%$ confidence level and a $5 \%$ margin of error. Non-adherence rates to ART regimen in India varies quite considerably from $14 \%-56 \%$ [9].

Records of 400 HIV patients out of 667 were selected by simple random sampling; if any record was found to be illegible, incomplete or damaged it was replaced by another record with registration number immediately following it. A predesigned, pretested and validated proforma was used for data collection from the Patient Treatment cards. Data was collected on patient demographics including probable route of HIV infection, date of HIV diagnosis, and ART history; clinical assessments, including data related to the occurrence of opportunistic infections and adverse events, laboratory data eg. CD4 cell count at time of enrollment to care, at the time months of date of survey), and past STIs diagnosed by clinic physicians, clinical profile, treatment adherence of the patients. An adherent patient was one who has not missed any dose of ART till date whereas a HIV patient who has missed one or more dose of ART was considered as non adherent patient for the present study. From the review of records the percentage of nonadherence was calculated among the study population (400) and then in the next phase all nonadherent persons (106) were interviewed over mobile phones to find out the cause of nonadherence. Interviewers received training in eliciting information on sensitive topics in a nonjudgmental manner. All data were compiled and analysed in Excel.Permission was taken from the Institution prior to data collection; Informed verbal consent was taken from the nonadherent patient population before the interviews through mobile phones.

\section{Result}

Table 1: Sociodemographic profile of study population $(\mathrm{n}=400)$

\begin{tabular}{|c|c|c|c|}
\hline Age groups & No. $(\%)$ & Education & No. $(\%)$ \\
\hline$<20$ & 12(3) & Illiterate & $143(35.83)$ \\
\hline $21-40$ & $271(67.9)$ & Primary \& secondary & $212(55.62)$ \\
\hline $40-60$ & $111(27.81)$ & College $\&$ above & $34(8.5)$ \\
\hline$>60$ & $5(1.25)$ & & \\
\hline Sex & No.(\%) & Occupation & No.(\%) \\
\hline Male & $279(73.47)$ & Employed & $272(68)$ \\
\hline Female & $113(28.31)$ & Unemployed/retired & $128(32)$ \\
\hline Transgender/transexual & $7(1.75)$ & & \\
\hline Residence & No. $(\%)$ & Income (PCMI in Rs.) & No. $(\%)$ \\
\hline Urban & $330(82.5)$ & $<773$ & $140(35)$ \\
\hline Rural & $70(17.5)$ & $773-1546$ & $126(31.5)$ \\
\hline & & $1547-2576$ & $58(14.5)$ \\
\hline & & $2577-5155$ & $41(10.2)$ \\
\hline & & $>5155$ & $35(8.75)$ \\
\hline
\end{tabular}

Majority (67.9\%) of the study population belonged to the age group of 21-40 years, $73.47 \%$ were males.82.5\% lived in urban area,64.17\% were literate, $(68 \%)$ were employed and 35\% had PCMI <Rs. $773 /-$. 
Table 2.Distribution of study population according to Clinical Profile $(n=400)$

\begin{tabular}{|l|l|}
\hline CD4 count & No.(\%) \\
\hline$<200 /$ cu.mm & $118(29.5)$ \\
\hline$>200 /$ cu.mm & $282(70.5)$ \\
\hline & \\
\hline Opportunistic infections & $178(44.5)$ \\
\hline Present & $222(55.5)$ \\
\hline Absent & \\
\hline Side effects of treatment & $78(19.5)$ \\
\hline Present & $322(80.5)$ \\
\hline Absent & \\
\hline
\end{tabular}

$70.5 \%$ of the study population had CD4 T cell count $>200$ cells/cu.mm, $44.5 \%$ of the study population manifested opportunistic infection, $80 \%$ showed no side effects of ART.

Fig.1 Distribution of study population according ART adherence $(n=400)$

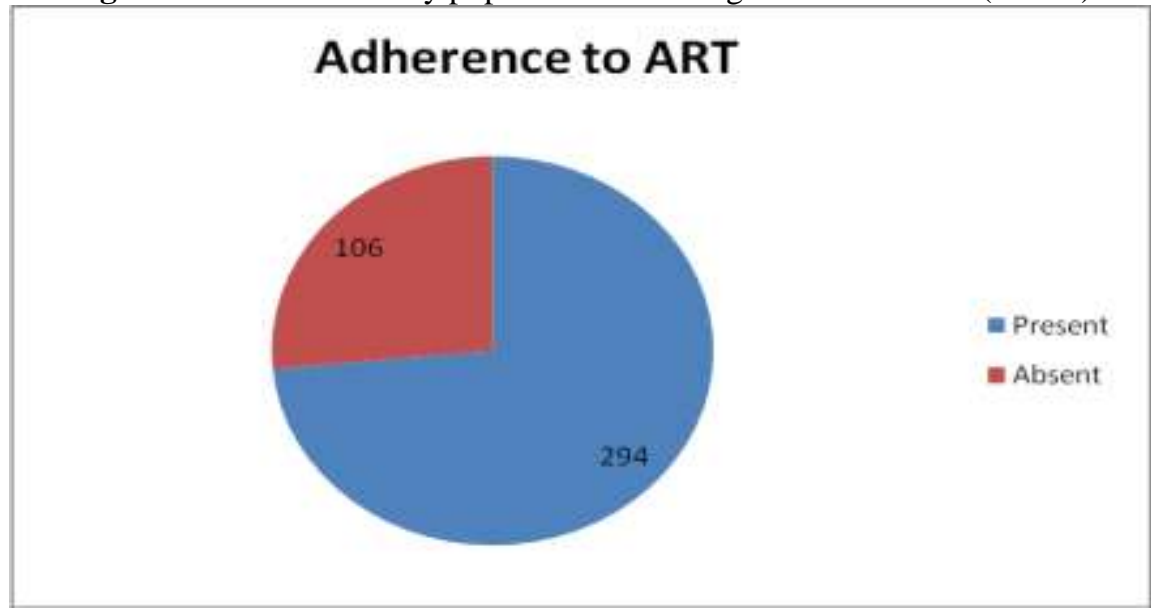

$26.5 \%$ of the study population were not adherent to ART therapy.

Fig.2 Distribution of study population according to cause of non adherence to ART ( $\mathrm{n}=106)$

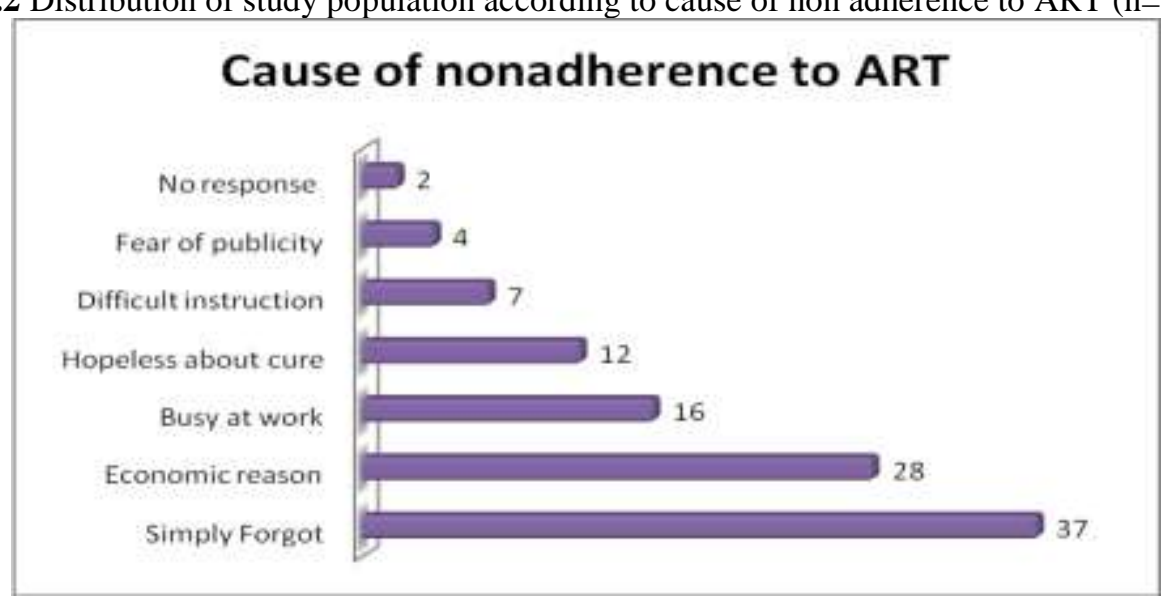

The findings from the present study suggest that although patients were motivated to register themselves at ART centres, constraints such as forgetfulness to take the medications (34.9\%),economic reason (26.4\%), busy at work (15.09\%), hopelessness about cure(11.32\%), difficult instructions for drug intake (6.6\%), fear of publicity $(3.77 \%)$ undermine their intentions to adhere to the ART regimen.

\section{Discussion}

Records of four hundred participants on ART regimen were reviewed in this study and those who were nonadherent were interviewed to find out the reasons. This research lays the groundwork for future interventions 
aimed at improving adherence. In the present study, $67.9 \%$ of the study population belonged to the age group of $21-40$ years, $73.47 \%$ were males, $64.17 \%$ of the study population were literate, $65 \%$ of the study population had PCMI > Rs. 773/-, 44.5\% had opportunistic infections. Similar findings was reported in a study by Rai \& Mahapatra, where $76 \%$ of the study participants were male, average 35 year old, $67 \%$ had formal education with median family income of 2000 INR per month, $50 \%$ of the patients had opportunistic infections at the time ART initiation. [8]

According to our study, $73.5 \%$ of the study population were adherent to ART. Our result is consistent with that of other similar studies conducted in India, where adherence ranges from $65 \%$ to $86 \%$ [10,11]. In the study by Karthik, Venkatesh et al $50.5 \%$ reported $100 \%$ HAART adherence[12]. In another study in Jharkhand $57 \%$ of the study participants were found to be adherent to ART regimen[8]. Cauldbeck reported $60 \%$ full adherence among the participants [13]. The difference in adherence level in the studies can be due to the different methods of adherence measurements like self-report approach, pill count approach etc.

Participants from the present study reported several constraints such as economic reason $(26.4 \%)$, forgetfulness to take the medications $(34.9 \%)$, difficult instructions for drug intake (6.6\%), busy at work (15.09\%), hopelessness about cure(11.32\%), fear of publicity (3.77\%) undermine their intentions to adhere to the ARV regimen. Forgetfulness was the most common cause of nonadherence in our study which can be attributed to the fact that AIDS in its late stages may affect the cognition of the patients. Participants complained about the cost of transport and other treatment-related costs incurred as a result of being on ARVs. Some reported taking their doses in private for fear that they would be discriminated against. Those who worked in private firms cited non-release by employers as a barrier to adherence to treatment. Treatment fatigue was common among those who were lacked self motivation, hopeless about cure and could not understand the instructions for drug use.

Venkatesh identified factors like side effects, socio-cultural factors, switch to alternative medicine system which influenced adherence [12]. According to Lal V, nonadherence was associated with not having been told about the importance of HAART, having to pay out-of-pocket for HAART [14]. A systematic review of 84 studies on adherence of ART across the world summarises the following important barriers for nonadherence- fear of disclosure, concomitant substance abuse, forgetfulness, suspicions of treatment, complicated regimens, work and family responsibilities and access to medication[15]. Kumarasamy et al. and Wanchu et al. showed that economic problems were barrier to ART adherence in India [16,17]. ART recipients from Indian and Sub Saharan African settings reported that factors such as forgetfulness, severity of adverse events and the level of complexity of the drug regimen, play an important role towards nonadherence [18].

We chose a retrospective cohort (nonadherent to ART) for telephonic interview to find out the cause of non adherence to ART; this could have introduced some amount of recall bias as the interview was conducted sometime after of their missed doses. But the strength of this study also lies in the fact that the nonadherent study participants directly expressed their views to the interviewers.

\section{Conclusion:}

Till now the ART program is running successfully in India, which is evident from the higher survival rate among ART adherent individuals. Under the programme, ART services are being provided to all HIV infected people free of cost. Still the high proportion of nonadherence to ART is a cause of concern as it may lead to the development of drug resistance. Our study may contribute towards the policy for improving adherence level in India by identifying the causes of nonadherence.

Efforts are required at the management as well as at implementation level for constant and regular follow-up with all patients under ART. Pill counting will help track the use of ARTs as well as help in calculating adherence rates throughout the country. In order to support ART users, more staff should be trained specifically in adherence counselling. As the disease progresses, some people who may not be able to attend the clinic, should receive a home visit for refills and counselling services. On the other hand there is a need to motivate the ART receivers by imparting knowledge about the benefits of ART. Awareness camps should be organized in the community to reduce stigma and discrimination associated with HIV. With these measures aimed at controlling nonadherence, ART programme in India can definitely accomplish its desired aim of making India AIDS free.

\section{Prof. P.K. Mitra, former director, IPGME\&R, Kolkata.}

\section{Acknowledgement}

\section{References}

[1]. Global report: UNAIDS report on the global AIDS epidemic 2013. "UNAIDS/ JC2502/1/E"- Revised and reissued, November 2013

[2]. Pandey, Geeta (2016-08-30). "The woman who discovered India's first HIV cases". BBC News. Retrieved 2016-12-08.

[3]. India HIV Estimates: Technical Report, 2015.(NACO )

[4]. Marfatia YS, Sharma A. Overview of HIV/AIDS in India .Indian Journal of Sex Transm Dis. 2007.Vol 28; No.1 
[5]. Wood E, Hogg RS et al. Effect of Medication Adherence on Survival of HIV-Infected Adults Who Start Highly Active Antiretroviral Therapy When the CD4+ Cell Count Is 0.200 to $0.350 \times 109$ cells/L. Annals of Internal Medicine.2003. 139: 810816

[6]. Oluwafemi O Oguntibeju. Quality of life of people living with HIV and AIDS and antiretroviral therapy. HIV AIDS (Auckl). 2012; 4: 117-124.

[7]. Kenneth MayerThe evolving Indian AIDS epidemic: Hope \& challenges of the fourth decade . Indian J Med Res. 2011 Dec; 134(6): 739-741.

[8]. Rai S, Mahapatra B. Adherence to Antiretroviral Therapy and Its Effect on Survival of HIV-Infected Individuals in Jharkhand, India. PLOS. June 18, 2013.

[9]. Mhaskar R, Alandikar V. Adherence to Antiretroviral Therapy in India: A Systematic Review and Meta-Analysis. Indian J Community Med. 2013 Apr-Jun; 38(2): 74-82.

[10]. Bachani D, Garg R, Rewari BB, Hegg L, Rajasekaran S, et al. Two-year treatment outcomes of patients enrolled in India's national first-line antiretroviral therapy programme. Natl Med J India.2010. 23: 7-12.

[11]. Sogarwal R, Bachani D .Assessment of ART centres in India: client perspectives. J Indian Med Assoc .2009.107: 276-280.

[12]. Venkatesh, K, Srikrishnan A. K. Predictors of Nonadherence to Highly Active Antiretroviral Therapy among HIV-Infected South Indians in Clinical Care: Implications for Developing Adherence Interventions in Resource-Limited Settings. AIDS Patient Care and STDs.2010. 24(12), 795-803

[13]. Cauldbeck M B, ${ }_{1}^{1} \mathrm{O}^{\prime}$ Connor C, ${ }^{2}$ O'Connor M. Adherence to anti-retroviral therapy among HIV patients

[14]. in Bangalore, India. AIDS Res Ther. 2009; 6: 7.

[15]. Lal V, Kant S, Dewan R, Rai SK, Biswas A. A two-site hospital-based study on factors associated with nonadherence to highly active antiretroviral therapy. Indian J Public Health .2010. 54: 179-183.

[16]. Mills EJ, Nachega JB, Bangsberg DR, Singh S, Rachlis B, et al. Adherence to HAART: a systematic review of developed and developing nation patient-reported barriers and facilitators. PLoS Med . 2006. 3: e438 10.1371/journal.pmed.0030438.

[17]. Kumarasamy N, Safren SA, et al. Barriers and facilitators to antiretroviral medication adherence among patients with HIV in Chennai, India: A qualitative study. AIDS Patient Care STDS 2005;19:526-37.

[18]. Wanchu A, Kaur R, Bambery P. Adherence to generic reverse transcriptase inhibitor-based antiretroviral medication at a Tertiary Center in North India. AIDS Behav. 2007;11:99-102

[19]. Mills EJ, Nachega JB, Buchan I et al. Adherence to antiretroviral therapy in sub-Saharan Africa and North America: A metaanalysis. JAMA. 2006;296:679-90. 